\title{
A Study of High-Frequency Regenerative Frequency Dividers
}

\author{
Amin Q. Safarian and Payam Heydari \\ Department of Electrical Engineering and Computer Science \\ University of California, Irvine \\ Irvine, CA 92697-2625
}

\begin{abstract}
A comprehensive analytical study of high-frequency regenerative frequency dividers (RFD) is presented. The study includes two fundamental modes of operation in RFDs, namely stable and pulled operation modes. Differential equations characterizing the RFD behavior for both operation modes are derived. Next, an RFD circuit is designed and simulated in a $0.18 \mu \mathrm{m}$ standard CMOS process. Simulations verify the accuracy of the proposed analytical models.
\end{abstract}

\section{INTRODUCTION}

Frequency dividers are ubiquitous building blocks used in a wide variety of important high-speed and radio-frequency (RF) integrated circuits, such as phase-locked loops (PLLs) and high-speed serializers/deserializers (SERDES). Introduced by Miller in 1939 [Mil39], a regenerative frequency divider (RFD) is essentially a non-linear feedback circuit consisting of a mixer and a loop-filter, as shown in Fig. 1.

In spite of having a simple steady-state operation, an RFD demonstrates complicated startup and transient operations. [Der91], [Har89], [Hel65] studied frequency-division criteria in an RFD, and showed that to establish a stable half-frequency regeneration two conditions must be satisfied (similar to oscillators). First of all, the loop gain at the half-frequency must be equal or greater than unity, and secondly, the total phase shift around the loop must be an integer multiple of $2 \pi$. [Adl46], [Raz03] introduced locking and pulling phenomena in oscillators. Studies undertaken by [Ad146], [Raz03] cannot be applied to RFDs, because the operation principle of RFDs is fundamentally different from that of oscillators. In contrast to injection-locked frequency dividers (ILFDs) [Dar89], [Rat99], there is no free running oscillation in RFDs. Moreover, the fed-back signal in an RFD is mixed with the input signal, as opposed to ILFDs in which the fed-back signal is added to the injected input signal. A comprehensive study of the RFDs is thus needed.

This paper presents an analytical study of RFDs. This study is applied to a commonly used example employing band-pass filters. The paper also includes the design and simulation of a regenerative frequency divider incorporating a distributed mixer circuit. The simulation results of the proposed RFD are then utilized to verify the accuracy of the analytical models.

The paper is organized as follows: Section 2 illustrates the architecture of RFD, and presents a comprehensive analytical study of stable and pulled operation of divider. Section 3 gives the simulation results validating the proposed equations. Finally, Section 4 provides the concluding remarks.

\section{REGENERATIVE FREQUENCY DIVIDER}

Depicted in Fig. 1 is the system block diagram of a general divide-by-two RFD. From a system-level perspective, RFD resembles a mixer-based PLL, but without the voltage-controlled oscillator (VCO).

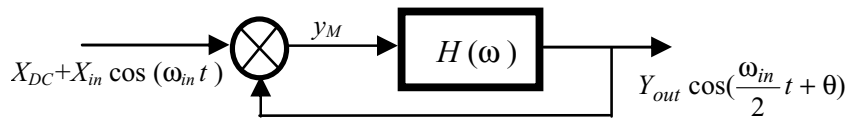

Fig. 1. The system block diagram of a divide-by-two regenerative frequency divider

The input signal and the fed-back output signal are the inputs to the constituent mixer depicted in Fig. 1. In steady state, the output signal of the mixer contains two harmonics at $\omega_{i n} / 2$ and $3 \omega_{i n} / 2$. The loop filter cancels out the frequency component at $3 \omega_{i n} / 2$. The output will thus run at half the input frequency.

The input to the high-frequency RFD is assumed to be a sinusoidal signal with an average value of $X_{D C}(c f$. Fig. 1) to include the commonly used signal waveforms used in RF systems. In the steady state, the output of the mixer, $y_{M}$, at the desired frequency, $\omega_{i n} / 2$, becomes (Note that $H(\omega)$ actually filters out the sum frequency component at $\left.3 \omega_{i n} / 2\right)$ :

$$
y_{M}=Y_{\text {out }}\left[X_{D C} \cos \left(\frac{\omega_{\text {in }}}{2} t+\theta(t)\right)+\frac{1}{2} X_{\text {in }} \cos \left(\frac{\omega_{\text {in }}}{2} t-\theta(t)\right)\right]
$$

where $\theta(t)$ is the time-varying phase-shift of the output signal to account for the time-varying phase-shift of the mixer. A time-varying phase-shift, $\theta(t)$, introduces harmonics at the output, and shifts the output frequency from its desirable half of the input frequency. According to Eq. (1), the output signal $y_{M}$ is comprised of two phasor components both running at $\omega_{i n} / 2$ with time-varying phase-shifts of $\pm \theta(t)$. The mixer output $y_{M}$, which is a phase-modulated (PM) signal, then passes through the loop filter. The behavior of a linear time-invariant (LTI) system in response to a PM (or FM) signal is, in general, complicated entailing approximate methods to evaluate the frequency spectrum of the PM signals and the response of an LTI system to the PM signals [Car02], [Pap02].

As will be illustrated in Section 2.B and Section 3, various experiments on high-frequency regenerative dividers show that the time-varying phase shift $\pm \theta(t)$ slowly varies with time. This observation helps us analyze the behavior of the loop filter in response to the phase-modulated $y_{M}$ signal by presenting the following theorem:

Theorem 1. Given an LTI system with the transfer function $H(\omega)$ and exposed to a phase modulated input signal of $\sin \left(\omega_{0} t+\theta(t)\right)$, if $\left|d^{k} \theta / d t^{k}\right|<\varepsilon$ for $k \geq 1$, the steady state output signal $y_{o}(t)$ can be written as:

$$
y_{o}(t)=\left|H\left(\omega_{0}+d \theta / d t\right)\right| \sin \left(\omega_{0} t+\theta(t)+\angle H\left(\omega_{0}+d \theta / d t\right)\right)
$$

$\underline{\text { Proof }}$

The proof is omitted due to the space limitation.

From another perspective, Theorem 1 states that the steady state response of an LTI system to a PM signal $\sin \left(\omega_{0} t+\theta(t)\right)$ with a slowly varying phaseshift $\theta(t)$, is similar to the steady state response of the system to a single tone $\sin \left(\omega_{0} t\right)$ with $\omega_{0}$ being replaced by the instantaneous frequency $\omega_{0}+d \theta / d t$. As a consequence, a slowly varying phase modulation around frequency $\omega_{0}$ is treated as a phase jitter in the frequency domain around $\omega_{0}$, i.e.,

$$
F\left\{e^{j\left(\omega_{0}+\theta(t)\right)}\right\} \approx \delta\left(\omega-\omega_{0}-\frac{d \theta(t)}{d t}\right)
$$

where $F\{$.$\} represents the Fourier transform. Utilizing Theorem 1, the$ output signal of the frequency divider after passing through the loop filter, $H(\omega)$, is written as follows:

$$
\begin{aligned}
Y_{\text {out }} \cos \left(\frac{\omega_{\text {in }}}{2} t+\theta\right) & =Y_{\text {out }} X_{D C}\left|H\left(\omega_{\text {in }} / 2+d \theta / d t\right)\right| \cos \left(\frac{\omega_{\text {in }}}{2} t+\theta(t)+\angle H\left(\omega_{\text {in }} / 2+d \theta / d t\right)\right) \\
& +\frac{1}{2} Y_{\text {out }} X_{\text {in }}\left|H\left(\omega_{\text {in }} / 2-d \theta / d t\right)\right| \cos \left(\frac{\omega_{\text {in }}}{2} t-\theta(t)+\angle H\left(\omega_{\text {in }} / 2-d \theta / d t\right)\right)
\end{aligned}
$$

Eq. (4) states that the output of an RFD in response to the PM signal at the input of the loop filter will be modulated both in the amplitude as well as the phase. A time-varying phase-shift associated with each constituent cosine function in (4) leads to an output signal whose amplitude and phase will be time-varying. Phasor algebra is utilized to obtain the closed-form time-domain expression for (4). The output signal of the frequency divider thus becomes: 


$$
\begin{aligned}
& Y_{\text {out }} \cos \left(\frac{\omega_{\text {in }}}{2} t+\theta(t)\right)= \\
& Y_{\text {out }} \frac{\left(X_{D C}+\frac{1}{2} X_{\text {in }}\right)\left|H\left(\omega_{\text {in }} / 2+d \theta / d t\right)\right| \cos \left[\theta(t)+\angle H\left(\omega_{\text {in }} / 2+d \theta / d t\right)\right]}{\cos \psi(t)} \cos \left(\frac{\omega_{\text {in }}}{2} t+\psi(t)\right)
\end{aligned}
$$

where $\psi(t)$ is the time-varying phase-shift of the mixer output $y_{M}$ (cf. Fig. $1)$ whose value is readily calculated:

$$
\psi(t)=\tan ^{-1}\left[\left(\frac{1+\frac{\alpha}{2}}{1-\frac{\alpha}{2}}\right) \tan \left(\theta(t)+\angle H\left(\omega_{i n} / 2+d \theta / d t\right)\right)\right]
$$

In Eq. (6), $\alpha=X_{i n} / X_{D C}$ is defined as the input power factor. Eq. (5) provides the loop equation of the RFD. Satisfying this equation sets forth the phase and amplitude criteria for correct division operation. As will be seen in Section 2.A., the phase relationship specifies the range of input frequency that guarantees the stable operation. It also determines the minimum required input signal to have a correct frequency division operation for any input frequency within the stable range of operation. Since the total phase-shift around RFD loop is frequency-dependent, the phase criterion is therefore particularly important. The phase condition also determines the output phase and instantaneous frequency in steady state. Equating the phase shifts of the right- and left-hand sides of Eq. (5) while considering (6) will lead to the following equation:

$$
\theta(t)=\psi(t)=\tan ^{-1}\left[\left(\frac{1+\frac{\alpha}{2}}{1-\frac{\alpha}{2}}\right) \tan \left(\theta(t)+\angle H\left(\omega_{i n} / 2+d \theta / d t\right)\right)\right]
$$

Eq. (7) presents a nonlinear differential equation (DE) for the RFD, characterizing the behavior of the RFD output phase with respect to other parameters including the phase shift of the loop filter. Further knowledge about the phase response of $H(\omega)$ is required prior to any discussion about solutions to Eq. (7). As an example which is particularly important in RF integrated circuits, we assume $H(\omega)$ to be a band-pass filter (BPF) whose amplitude and phase responses are even and odd functions of $\omega$, respectively. To attain guaranteed half-frequency regeneration in an RFD employing the BPF, the resonant frequency of the BPF at which the magnitude response of the BPF reaches its maximum, is set equal to $\omega_{i n} / 2$. To simplify (7) and obtain closed-form analytical model for phase-shift $\theta(t)$, we postulate that the loop BPF, $H(\omega)$, is realized using the commonly used $L C$ tank circuit [Jez74] with resonant frequency of $\omega_{0}$, and quality factor of $Q$. On the other hand, a slowly varying phase shift associated with the output of a high frequency RFD implies that the offset frequency is small compared to the output frequency of the RFD. Consequently, the phase analysis is carried out in the vicinity of $\omega_{0}$. Therefore, the phase response of the loop filter will approximately become:

$$
\begin{aligned}
\angle H(\omega) & =-\tan ^{-1} Q\left(\frac{\omega}{\omega_{0}}-\frac{\omega_{0}}{\omega}\right) \\
& \approx \tan ^{-1} \frac{2 Q}{\omega_{0}}\left(\omega_{0}-\omega\right)
\end{aligned}
$$

Using Theorem 1, the radian frequency, $\omega$, in Eq. (8) is replaced with its instantaneous value, $\omega_{i n} / 2+d \theta / d t$. Eq. (8) helps us approximate Eq. (7) with the following ordinary differential equation (ODE) (details are omitted due to the lack of space):

$$
\frac{d \theta}{d t}=\omega_{0}-\frac{\omega_{i n}}{2}-\frac{\omega_{0}}{2 Q} \frac{\frac{\alpha}{4} \sin 2 \theta}{1-\frac{\alpha}{2} \cos 2 \theta}
$$

The solution to the characteristic ODE in Eq. (9) is a periodic function of time, which means that the side-band frequency components around the main spectral line, $\omega_{i n} / 2$, due to $\theta(t)$ are equally spaced in the frequency domain.
According to (9), the RFD will acquire lock to the half-frequency if and only if $d \theta / d t$ becomes zero. Eq. (9) makes it possible to distinguish between two modes of operation in an RFD, namely stable and pulled operation. As will be observed in Section 2.A., in the stable mode, the output frequency is time-independent; therefore, the output spectrum is pure and free of spurs. On the contrary, during the pulled operation, the output phase shift, $\theta(t)$, at the output varies with time while introducing a small frequency offset to $\omega_{i n} / 2$ and spurs in the output spectrum.

Interestingly, Eq. (11) is similar in form to an equation derived earlier by Adler [Adl46] to characterize the locking phenomenon in freerunning oscillators. However, in contrast to free-running oscillators, in which $\sin \theta$ appears in the characteristic differential equation under injection locking, for the regenerative frequency dividers the rate of change of the output phase is a function of input power factor, $\alpha$, and $2 \theta$.

\section{A. Stable Operation}

By definition, RFD has a correct and stable frequency division, if only if the instantaneous output frequency, $\omega_{i n} / 2+d \theta / d t$, does not change with respect to time (i.e., $d \theta / d t=0$ ). Eq. (9) will become:

$$
A \sin ^{2} 2 \theta+B \sin 2 \theta+C=0
$$

where

$$
\begin{aligned}
& A=\frac{\alpha^{2}}{4}\left(1+\left(\frac{\omega_{0}}{2 Q} \frac{1}{2 \Delta \omega_{i}}\right)^{2}\right) \\
& B=-\left(\frac{\alpha}{2}\right)\left(\frac{\omega_{0}}{2 Q}\right)\left(\frac{1}{\Delta \omega_{i}}\right) \\
& C=1-\frac{\alpha^{2}}{4}
\end{aligned}
$$

In the stable mode, the characteristic ODE of the RFD simply becomes an algebraic equation. As will be seen later in this section, Eq. (10) will specify three underlying attributes of an RFD in its stable operation regime:

(I) The input frequency range, or lock range, $\Delta \omega_{i}=\omega_{0}-\omega_{i n} / 2$, which guarantees the stable operation of the RFD.

(II) The value of the output phase $\theta(t)$ at a given input frequency within stable operation range.

(III) The minimum required input power factor, $\alpha$, in order to have frequency division in the stable mode.

Any real solution of the second-order algebraic equation whose absolute value is less than unity (i.e., $\sin 2 \theta=\left|f\left(\alpha, \Delta \omega_{i}\right)\right| \leq 1$ ) is considered as a valid solution for Eq. (10). Having obtained a valid solution for Eq. (10) means that the left-hand side of the ODE given by Eq. (9) is zero, i.e., $d \theta / d t=0$, which in turn means that the RFD is in the stable mode.

The root pair of Eq. (10) resides in $(-1,1)$, if and only if:

$$
B^{2}-4 A C \geq 0 \text { and }-1 \leq-\frac{B}{2 A} \leq 1
$$

Equations (10) and (12) result in $-1 \leq C / A \leq 1$, or:

$$
\left|\Delta \omega_{i}\right| \leq \frac{\omega_{0}}{4 Q} \sqrt{\frac{\alpha^{2}}{1-\frac{\alpha^{2}}{2}}}
$$

Eq. (13) specifies the range of input frequencies at which the RFD system operates in stable operation. Moreover, rearranging (13) with respect to $\alpha$ will result in an analytical closed-form expression for the minimum required input to achieve the half-frequency regeneration:

$$
\alpha \geq \frac{1}{\sqrt{\frac{1}{2}+\left(\frac{\omega_{0}}{2 Q}\right)^{2} \frac{1}{\left(2 \Delta \omega_{i}\right)^{2}}}}
$$

Eq. (14) characterizes an important attribute of the RFD, namely the input sensitivity. More precisely, this equation states that for a given loop filter the minimum required input to achieve the frequency regeneration increases with the offset frequency. The minimum required input will 
achieve its lower limit if the center frequency of the loop filter is tuned at exactly the half-frequency.

\section{B. Pulled Operation}

If a valid solution for Eq. (10) does not exist, the output frequency then deviates from its desired half of input frequency. In other words, the lefthand side of the characteristic ODE cannot be zero (i.e., $d \theta / d t \neq 0$ ). A time varying phase, $\theta(t)$, at the output causes a deviation of the output frequency from its desired value. The RFD is thus in the pulled operation mode. One important phenomenon causing the RFDs to operate in the pulled mode might be the deviation of resonant frequency of the constituent loop filter, $\Delta \omega_{0}$, due to the process variation. For instance, if the $\mathrm{k}^{\text {th }}$ metal layer used to implement the passive elements of the loop filter experiences a width variation of $\Delta w_{k}$ and a height variation of $\Delta h_{k}$ due to the process variation of the interlayer dielectric, the resistance, capacitance, and inductance of the loop filter will experience offsets, accordingly. Offsets associated with the passive elements directly contribute to small variation of the resonant frequency.

In the pulled operation mode, the characteristic ODE in Eq. (9) must be solved directly to obtain the time-varying phase-shift $\theta(t)$. The general solution to Eq. (9) is, however, too complicated. To gain an insightful knowledge about the RFD behavior, the analysis is simplified for two special cases: (1) $\alpha<<1$; and (2) $\alpha>>1$.

For small input power $(\alpha<<1)$, the ODE in (9) becomes:

$$
\frac{d \theta}{d t} \approx \omega_{0}-\frac{\omega_{i n}}{2}-\frac{\omega_{0}}{2 Q} \frac{\alpha}{4} \sin 2 \theta
$$

A general solution of Eq. (15) is as follows:

$$
\theta(t)=\tan ^{-1}\left(\frac{\omega_{L}}{\Delta \omega_{i}}+\frac{\omega_{S}}{\Delta \omega_{i}} \tan \omega_{S} t\right)
$$

where $\omega_{S}=\sqrt{\Delta \omega_{i}^{2}-\omega_{L}^{2}}$, for pulled operation: $\left|\Delta \omega_{i}\right|>\omega_{L}$

As expected, the time-varying phase-shift at the output of the RFD $\theta(t)$ is a periodic phase with the radian frequency of $\omega_{S}$, where $\omega_{S}$ is smaller than $\omega_{0}$. Eq. (15) quantifies another foregoing observation, that is, the spectrum of the output signal contains a fundamental component not exactly at $\omega_{i n} / 2$, but deviated from that, plus an infinite number of sideband spurs that are equally spaced by the radian frequency of $\omega_{S}$ during the pulled operation. A similar phenomenon is seen in narrow-band FM signals [Carl02], which is observed in simulation results, too (see Figs. 6 (a)-(e)). As input signal power to the RFD increases, the frequency spacing between spurs will be reduced, and eventually the RFD will become stable.

For large-signal input amplitudes (i.e., $\alpha>>1$ ), the ODE in Eq. (9) is simplified as follows:

$$
\frac{d \theta}{d t} \approx \Delta \omega_{i}+\frac{\omega_{0}}{4 Q} \tan 2 \theta
$$

The right-hand side of Eq. (18) includes $\tan 2 \theta$, which means that there always exists a value for $\theta(t)$ which makes $d \theta / d t$ zero. This also means that if the RFD is initially in the pulled operation mode, it will attain the stable mode for sufficiently large input signal even in the presence of the process variation. In fact, the output phased-shift $\theta(t)$ in the stable mode is specified as follows:

$$
\theta(t)=-\frac{1}{2} \tan ^{-1}\left(\Delta \omega_{i}+\frac{\omega_{0}}{4 Q}\right)
$$

For an invariable input frequency and loop filter, $\theta(t)$ will no longer be time-variant, and the RFD is in stable operation mode.

What can be said about the RFD behavior for the moderate input amplitudes? For the moderate values of the input amplitudes, so long as the input power factor satisfies Eq. (14), the RFD will be operating in the stable operation mode. Otherwise, it will be in the pulled operation region. This phenomenon was also observed in actual simulations of the RFD frequency synthesizer.

\section{EXPERIMENTAL RESULTS}

To verify the proposed analytical models developed for the RFD in the stable and the pulled operation modes, a CMOS RFD is designed in a 0.18 $\mu \mathrm{m}$ standard CMOS process. The RFD employs a CMOS distributed mixer proposed in [Saf04] where each cell is realized using a single balanced mixer, as shown in Fig. 2.

In a distributed mixer the single balanced cells are distributed along the artificial LC transmission lines. The designed mixer circuit incorporates two-stage architecture. Transmission lines are realized using LC ladder circuits ${ }^{\mathrm{T}}$. Five distinct RF, LO, and IF artificial lines are employed in the circuit. The parasitic gate and drain capacitances along with the inductors constitute the artificial transmission lines. Post-layout simulations are carried out to account for the metal and interconnect parasitics.

The RFD is designed to operate at an input frequency of $40 \mathrm{GHz}$. The inductors $L_{R F}, L_{L O}$ and $L_{I F}$ are $1 \mathrm{nH}$. The termination impedances $Z_{R F}, Z_{L O}$, and $Z_{I F}$ are $50 \Omega$. An LC band-pass filter with the resonant frequency of 20 $\mathrm{GHz}$ is used as the loop filter, $H(\omega)$. The bias current $I_{D C}$ is set at 2.8, 3.8 and $4.8 \mathrm{~mA}$ to investigate the sensitivity of the RFD for different values of the current tail.

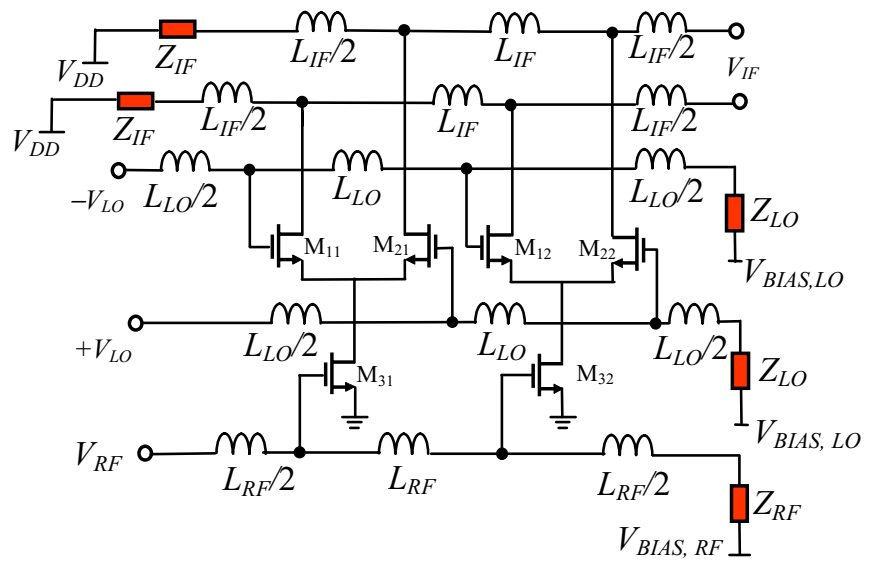

Fig. 2. Distributed single balanced mixer used in the proposed RFD

Fig. 3 indicates the minimum required input power vs. the input frequency offset, $\Delta \omega_{i}$, from the center frequency of $40 \mathrm{GHz}$. A comparison is made between the HSPICE simulation and the analytical model presented in Eq. (14). Fig. 3 also shows the stable and pulled operation regions of the RFD. As seen in Fig. 3, the analytical derivation of Eq. (14) closely follows the simulation result. Fig. 4 demonstrates the simulated input and output waveforms of the frequency divider. The input frequency is $40 \mathrm{GHz}$, while the output is locked at $20 \mathrm{GHz}$. Depicted in Fig. 5 is the minimum required input power under the three different tail currents. Solid lines show the analytical derivation of Eq. (14). It is evident from Fig. 5 that increasing the bias current requires more input power to achieve the stable operation, thereby confirming Eqs. (13) and (14).

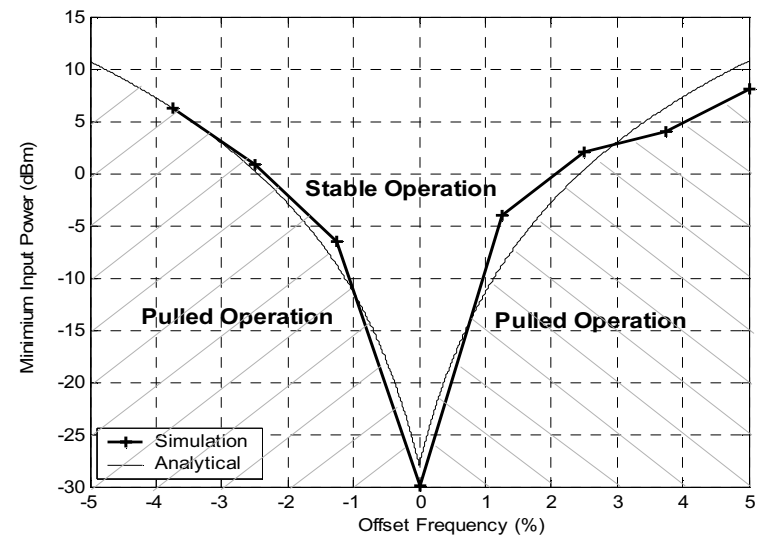

Fig. 3. Stable and pulled operation of RFD

${ }^{1}$ Another alternative is to use on-chip micro-strip lines. 


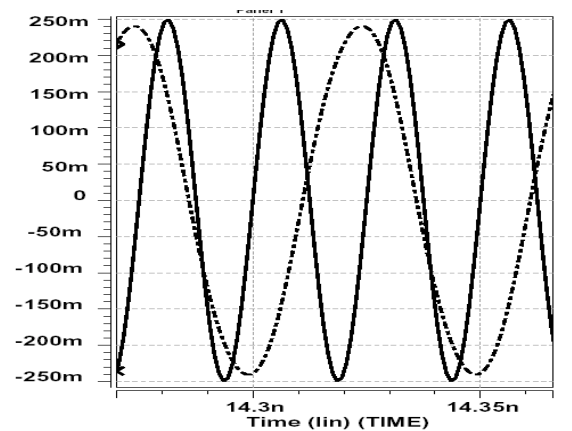

Fig. 4. Input (solid) and output (dashed) waveforms

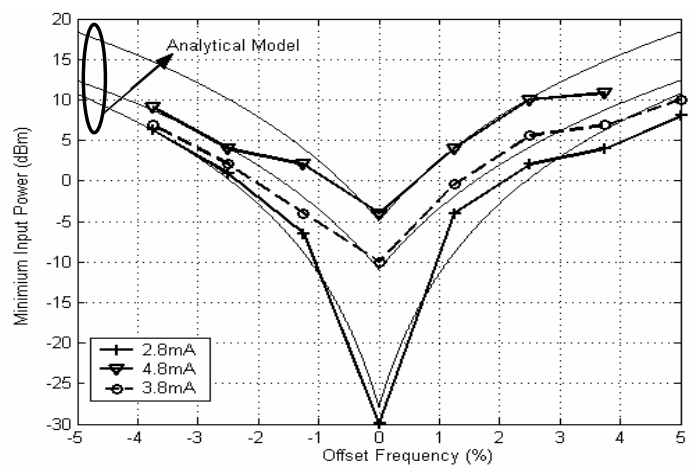

Fig. 5. Minimum required input vs. Offset frequency

As an example to demonstrate the pulled operation, the inductance value is varied by up to $10 \%$ to obtain a variation of $\Delta \omega_{0} / \omega_{0}=5 \%$ for the $L C$ tank resonant frequency (because $\left|\Delta \omega_{0} / \omega_{0}\right|=|\Delta L / 2 L|$ ). The RFD is locked to $\omega_{i n} / 2=20 \mathrm{GHz}$ for the small input amplitudes. Simulation results in Figs. 6 (a)-(e) show that this variation in resonant frequency has some effects on the output signal of the RFD: (1) output frequency deviates by $\Delta \omega_{\text {out }} \approx \omega_{S}$ $\left[\omega_{S}\right.$ is defined by Eq. (17)] from its desired value, $\omega_{i n} / 2,(2)$ symmetric spurs generate in the output spectrum, (3) spurs are located at $\Delta \omega_{\text {out }}$ of each other.

Meanwhile, as the input signal power increases, the spurs become closer to each other, and also to the desirable output frequency, $\omega_{i n} / 2$. Furthermore, the number of spurs increases. These observations are all verified by equations (16), and (17) that include the definitions for $\omega_{i n}, \theta(t)$, and $\omega_{S}$. It seems that the average output power will be distributed over a finite bandwidth around the half-frequency component. Increasing the input signal amplitude causes the spurs to get closer to each other; hence more spurs will appear around of the output spectral line (cf. Figs. 6 (a)-(e)). This phenomenon will continue until the input signal exceeds a limit specified by Eq. (14). After that limit all the spurs disappear and the small frequency deviation of output signal from its desired value becomes zero. In other words, $\Delta \omega_{0}$ causes a deviation in output frequency from $\omega_{i n} / 2$ or an increase in minimum required voltage for correct division. Solutions to Eq. (9) in the presence of deviation of the resonant frequency of the loop filter will give us the corresponding analytical model of the phenomena specified above. Therefore, for small input power factors, deviation in the $L C$ tank resonant frequency causes the RFD to operate in the pulled operation region, shift the output frequency from its desired value, and generate symmetric spurs.

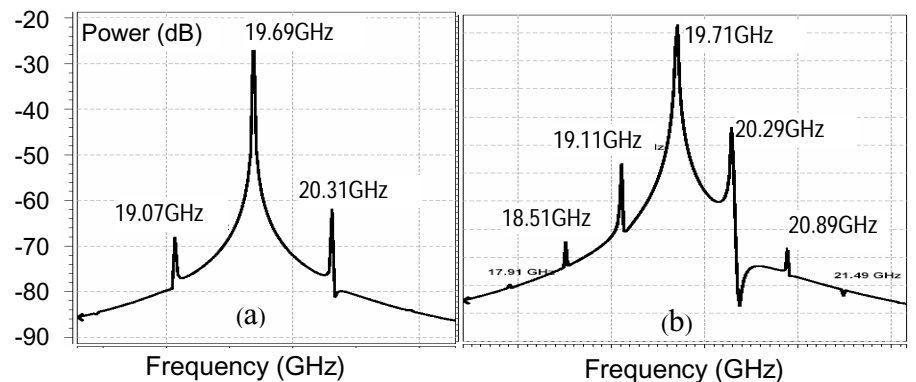

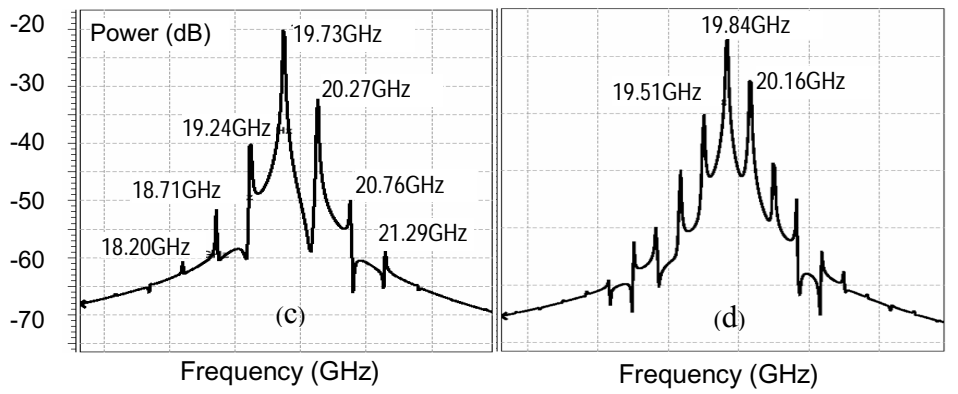

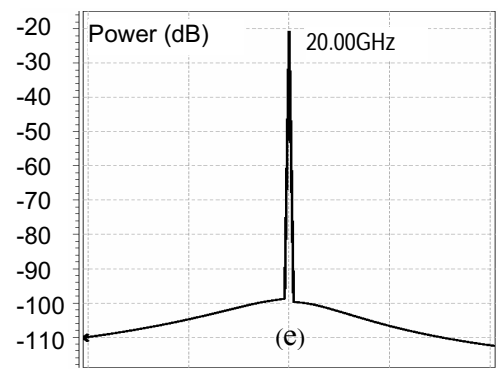

Frequency $(\mathrm{GHz})$

Fig. 6. Output spectrum for different input powers in the pulled mode operation. The input power factor is increasing from its lowest value in (a) to the largest value in (e)

\section{CONCLUSIONS}

In this paper an analytical study of regenerative frequency dividers (RFD) was presented. Two modes of operation; stable and pulled, were studied and the characteristic differential equations (ODE) expressing the RFD behavior of these two modes were derived. An RFD was realized utilizing a CMOS distributed mixer. Observation from simulation results validated the developed analytical models for both modes of operation.

\section{Acknowledgements}

The authors would like to thank Broadcom, Inc., for their support and Jazz Semiconductor, Inc., Newport Beach, CA for providing the device and simulation data, and in particular, Marco Racanelli, Paul Colestock for their help and support.

\section{REFERENCES}

[Adl46] R. Adler, "A Study of Locking Phenomena in Oscillators," Proc. IRE, vol. 34, pp. 351-357, June 1946.

[Car02] A. B. Carlson, et. al., Communication Systems, McGraw Hill, Fourth Edition, 2002

[Dar89] A. S. Daryoush, T. Berceli, R. Saedi, P. R. Herczfeld, A. Rosen, "Theory of Subharmonic Synchronization of Nonlinear Oscillators," IEEE MTT-S, vol. 2, pp. 735-738, June 1989.

[Der91] R. Derksen, et. al,"Stability Ranges of Regenerative Frequency Dividers Employing Double Balanced Mixers in Large Signal Operation," IEEE Transaction of MTT, Vol.39, No.10, Oct. 1991

[Har89] R. Harisson, "Theory of Regenerative Frequency Dividers using DoubleBalanced Mixers", IEEE Digest MTT-S, 1989

[Hel65] C. Helstrom, "Transient Analysis of Regenerative Frequency Divider", IEEE Transaction on Circuit Theory, vol. 12, no. 4, Dec. 1965

[Jez74] M. Jezewski, "An approach to the analysis of injection-locked oscillators", IEEE Trans. on Circuits and Systems, Vol. 21, no. 3, pp. 395 -401, May 1974.

[Mil39] R. L. Miller, "Fractional-frequency Generators Utilizing Regenerative mModulation", Proc. IRE, pp. 446-457, vol.27, Jul 1939.

[Pap02] A. Papoulis, et. al, Probability, Random Variables and Stochastic Processes, Mc-Graw Hill, $4^{\text {th }}$ Edition, 2002.

[Rat99] H. R. Rategh and T.H. Lee, "Superharmonic Injection-Locked Frequency Dividers," IEEE J. Solid-State Circuits, vol. 34, pp. 813-821, June 1999.

[Raz03] B. Razavi, "A Study on Locking and Pulling of Oscillators Under Injection," IEEE CICC, pp. 305-213, May 2003.

[Saf04] A. Safarian and P. Heydari, " Design and and Analysis of a Distributed Regenerative Frequency Divider Using a Distributed Mixer," to appear in IEEE International Symposium on Circuits and Systems, May 2004. 